\title{
EFECTO DE DOS ANTIOXIDANTES (TEMPO Y TEMPOL) EN LA CRIOPRESERVACIÓN DE SEMEN OVINO EMPLEANDO UN DILUTOR EN BASE A TRIS
}

\author{
Effect of Two Antioxidants (Tempo and Tempol) on Ram Semen \\ Cryopreservation Using the Tris Extender
}
Luis Ruiz G. ${ }^{1,4}$, Alexei Santiani A. ${ }^{1}$, Rocío Sandoval M. ${ }^{1}$, Wilfredo Huanca L. ${ }^{1}$, Alfredo Delgado C. ${ }^{2}$, Luis Coronado S. ${ }^{3}$ y César Alzamora P. ${ }^{3}$

\section{Resumen}

\begin{abstract}
Se emplearon 32 muestras de semen procedentes de cuatro ovinos a fin de ser criopreservadas con la adición dos antioxidantes: Tempo (2,2,6,6 tetrametil-1-piperidiniloxil) y Tempol (4-hidroxi 2,2,6,6 tetrametil-1-piperidiniloxil), en concentraciones de 0.5, 1.0 y 2.5 $\mathrm{mM}$, para evaluar el efecto postdescongelamiento que pudieran tener sobre la motilidad progresiva, la viabilidad e integridad acrosomal y la capacitación espermática prematura. En cada concentración de los dos antioxidantes y del grupo control se utilizó un dilutor en base a Tris, realizándose ocho repeticiones, cada una con cuatro muestras de semen. Los resultados obtenidos demuestran que la adición de Tempo a una concentración de $0.5 \mathrm{mM}$ mejora significativamente la calidad del semen criopreservado en comparación con el grupo control ( $\mathrm{p}<0.05)$, incrementado los porcentajes de motilidad progresiva (79 vs. $67 \%)$, viabilidad e integridad acrosomal (70 vs. $58 \%$ ) y reduciendo la capacitación espermática prematura (9 vs.15\%). Por otro lado, la adición de Tempol disminuyó la calidad seminal postdescongelamiento en comparación con el control. En conclusión, la adición de Tempo $0.5 \mathrm{mM}$ en un dilutor en base a Tris, al finalizar la fase de enfriamiento, podría constituir una estrategia para mejorar la calidad de semen ovino criopreservado.
\end{abstract}

Palabras clave: antioxidante, Tempo, Tempol, criopreservación, semen, ovino, Tris

\section{Abstract}

Thirty-two semen samples from four rams were frozed using two antioxidants, Tempo (2,2,6,6 tetramethyl-1-piperidinyloxyl) and Tempol (4-hidroxi 2,2,6,6 tetramethyl-1piperidinyloxyl), each one in concentrations of $0.5,1.0$, and $2.5 \mathrm{mM}$, to evaluate the effects on post-thawing motility, viability, acrosomal integrity, and earlier sperm capacitation. Eight replications for each antioxidant concentration and control group,

\footnotetext{
${ }^{1}$ Laboratorio de Reproducción Animal, ${ }^{2}$ Clínica de Animales Mayores, ${ }^{3}$ Laboratorio de Producción Agropecuaria, Facultad de Medicina Veterinaria, Universidad Nacional Mayor de San Marcos, Lima

${ }^{4}$ E-mail:frg81@hotmail.com
} 
were done using four semen samples per replication. Semen samples were diluted on a Tris extender. Results showed that Tempo $0.5 \mathrm{mM}$ improved frozen semen quality in comparison with control group ( $\mathrm{p}<0.05)$, by increasing progresive motility (79 vs. $67 \%$ ), viability and acrosomal integrity (70 vs. 58\%), and decreasing earlier sperm capacitation (9 vs. 15\%). On the other hand, Tempol decreased frozen semen quality. In conclusion, the use of Tempo $0.5 \mathrm{mM}$ on a Tris extender, at the end of the cooling process, could be an alternative for improving the quality of frozed ram semen.

Key words: antioxidants, Tempo, Tempol, criopreservation, semen, ram, Tris

\section{INTRODUCCIÓN}

El proceso de criopreservación de semen en ovinos causa una disminución de la motilidad y viabilidad espermática, así como cambios relacionados a la capacitación espermática. Estas alteraciones producen una disminución de la capacidad fecundante del semen criopreservado, por lo que al ser usado se obtienen bajas tasas de concepción (Watson, 2000). Las alteraciones son parcialmente producidas por el incremento en la producción de especies reactivas de oxígeno durante el proceso de criopreservación, causando daños oxidativos en las biomoléculas que componen al espermatozoide, como peroxidación lipídica, oxidación de las proteínas y daños en el ADN (Aitken y Fisher, 1994). Esto ha sido evidenciado en humanos (Wang et al., 1997), bovinos (Chatterjee y Gagnon, 2001) y ovinos (Santiani, 2003). En estos últimos se ha encontrado que la producción de especies reactivas de oxígeno ocurre principalmente durante el periodo de enfriamiento, alcanzando valores máximos cuando el semen es mantenido a $5{ }^{\circ} \mathrm{C}$ (Santiani, 2003).

Normalmente, las especies reactivas de oxígeno son producidas en la mitocondrias, durante el consumo de oxígeno, cuando el agua se reduce secuencialmente. En el espermatozoide ovino y de otros rumiantes se ha encontrado que se producen por la desaminación oxidativa de los aminoácidos aromáticos (Upreti et al., 1998). Durante la criopreservación de semen, su incremento se explica por la presencia de sustancias prooxidantes en los dilutores (Bilodeau et al., 2002). El espermatozoide ovino es muy susceptible a la acción de las especies reactivas de oxígeno, ya que su membrana plasmática presenta una alta cantidad de fosfolípidos (Davis, 1981) que contienen principalmente ácidos grasos insaturados, predominando el ácido docosahexaenoico que es un ácido graso de cadena larga de 22 carbonos y 6 dobles enlaces (Jones y Mann, 1977). También es susceptible por ser pobre en colesterol y tener una menor relación colesterol-fosfolípidos que otras especies, permitiendo que la capacitación espermática ocurra más rápido que en otras especies (Davis, 1981).

En situaciones normales, el semen presenta sustancias de capacidad antioxidante. Estos antioxidantes se encuentran en los espermatozoides y en el plasma seminal, existiendo una interacción y sinergismo entre ellos. En el semen ovino se ha encontrado SOD, catalasa, glutation peroxidasa, vitamina C y E y ácido úrico (Ochsendorf, 1997). Estos antioxidantes tienen la función de prevenir el daño oxidativo. Durante la criopreservación de semen, el descenso de la temperatura produce una disminución de la actividad y de la concentración de las enzimas antioxidantes (Álvarez y Storey, 1992).

En los últimos 25 años se ha encontrado que la adición de antioxidantes a los dilutores (? -tocoferol, hidroxitolueno butilado, taurina, EDTA y análogos de la superóxido dismutasa) durante el proceso de criopreservación reduce el daño oxidativo (Watson y Anderson, 1983; Askari et al., 
1994; Sánchez-Partida et al., 1997; Aisen et al., 2000; Santiani, 2003). Así mismo, antioxidantes como ácido ascórbico (Askari et al., 1994), hipotaurina y carnosina (SánchezPartida et al., 1997) no mejoran la función espermática luego del proceso de criopreservación.

En 1990 se descubrió que el Tempo (2,2,6,6 tetrametil-1-piperidiniloxil) y Tempol (4-hidroxi 2,2,6,6 tetrametil-1-piperidiniloxil) tenían actividad similar a la SOD (Mitchell et $a l .$, 1990). Éstos pertenecen a un grupo de radicales nitróxidos estables, con la ventaja de tener un bajo peso molecular, una mayor permeabilidad, ser independientes de metales, tener una mayor vida metabólica y no ser consumidos durante las reacciones (Luo, 2001).

El Tempo y Tempol han sido utilizados con la finalidad de prevenir la pérdida de motilidad y viabilidad espermática durante la refrigeración de los espermatozoides de ovino (Mara et al., 2005), equino (Ball et al., 2001), y pavo (Donogue y Donogue, 1997), así como durante el congelamiento de espermatozoides de ovino (Santiani, 2003) y bovino (Foote et al., 2002). Se ha encontrado que la adición de Tempo o Tempol a un medio en base a Tris en la criopreservación de semen bovino no afectó la motilidad espermática, y cuando se usó leche descremada como dilutor, la motilidad disminuyó significativamente (Foote et al., 2002), demostrando que los efectos del empleo de estos antioxidantes dependen del dilutor en que se encuentren. Su adición al semen ovino en un medio en base a leche descremada estarían previniendo parcialmente la pérdida de motilidad y viabilidad, así como reduciendo la proporción de espermatozoides capacitados prematuramente (Santiani, 2003); sin embargo, en trabajos previos en el mismo laboratorio se obtuvieron mejores resultados con un medio en base a Tris (Sandoval, 2005).
Por lo tanto, el objetivo del presente trabajo fue evaluar el efecto de la adición de tres concentraciones de los antioxidantes Tempo y Tempol sobre la calidad de semen post-descongelamiento en un dilutor en base a Tris.

\section{Materiales y Métodos}

\section{Lugar de estudio y animales}

El estudio se realizó en el Laboratorio de Reproducción Animal de la Facultad de Medicina Veterinaria de la Universidad Nacional Mayor de San Marcos (FMVUNMSM). La colección de semen se realizó con vagina artificial a dos carneros Merino y dos Pelibuey, de dos y tres años de edad, en forma semanal y por ocho semanas.

Se procesaron únicamente aquellas muestras con un volumen $>0.8 \mathrm{~mL}$, motilidad masal ?4 y una concentración $>1000 \times 10^{6}$ espermatozoides $/ \mathrm{mL}$. Los eyaculados se mezclaron para obtener una única muestra por semana.

\section{Procedimiento metodológico}

Se evaluó el efecto de la adición de los antioxidantes Tempo ${ }^{5}(2,2,6,6$ tetrametil-1piperidiniloxil) y Tempol $^{6}$ (4-hidroxi 2,2,6,6 tetrametil-1-piperidiniloxil), en tres diferentes concentraciones $(0.5,1.0$ y $2.5 \mathrm{mM})$ sobre la calidad del semen criopreservado. Además, se dejó un grupo control sin antioxidante.

\section{Método de congelamiento}

El pool de semen se mezcló con la primera fracción del diluyente (1:1) previamente estabilizado a $35^{\circ} \mathrm{C}$ en baño maría. La primera fracción del diluyente fue preparada con tris $27.1 \mathrm{~g}$, ácido cítrico $14 \mathrm{~g}$, fructosa

\footnotetext{
${ }^{5}$ Aldrich (214000)

${ }^{6}$ Sigma $(H-8258)$
} 
$10 \mathrm{~g}$ y yema de huevo $10 \%$, diluidos en agua bidestilada csp. 1 L (Aisen et al., 2000). El semen diluido fue colocado en un tubo falcón de $15 \mathrm{~mL}$ y llevado a baño maría $\left(35^{\circ} \mathrm{C}\right)$ para iniciar la curva de enfriamiento.

El enfriamiento se realizó utilizando agua fría, a una velocidad aproximada de $1^{\circ} \mathrm{C} / 3$ min desde los $35{ }^{\circ} \mathrm{C}$ hasta los $5{ }^{\circ} \mathrm{C}$. Los antioxidantes se agregaron cuando la temperatura llegó a $10^{\circ} \mathrm{C}$. A los $5^{\circ} \mathrm{C}$ se agregó igual volumen de la segunda fracción del diluyente (glicerol $6 \%$, trehalosa $76 \mathrm{~g}$ y EDTA $1.5 \mathrm{~g}$, diluidos en la primera fracción csp. 1 L), previamente enfriado a la misma temperatura, obteniendo una concentración final de 300 a 400 millones de espermatozoides/mL. Se dejó estabilizar por media hora y se procedió a envasar con ayuda de una jeringa en pajillas de $0.5 \mathrm{~mL}$. Estas fueron congeladas con los vapores de nitrógeno líquido. Se disminuyó la temperatura de $5 \mathrm{a}-25{ }^{\circ} \mathrm{C}$ en un lapso de 6 minutos $\left(5^{\circ} \mathrm{C} / \mathrm{min}\right)$ y finalmente, fueron sumergidas en el nitrógeno líquido.

\section{Evaluación del semen congelado}

El descongelamiento se realizó colocando cada pajuela en baño maría $\left(37^{\circ} \mathrm{C}\right)$ por 10 s. Luego, el semen se diluyó a razón de 1 en 10 en un medio base (primera fracción del dilutor sin yema de huevo) a $37{ }^{\circ} \mathrm{C}$, y luego de 20 min se evaluó la motilidad progresiva, la viabilidad e integridad del acrosoma y la capacitación espermática.

Para evaluar la motilidad progesiva, el semen se diluyó en suero fisiológico temperado a $37^{\circ} \mathrm{C}$ y se observó en un microscopio óptico a 400x calculándose el porcentaje de espermatozoides con movimiento hacia delante.

La evaluación de la viabilidad e integridad acrosomal se realizó mediante la técnica de doble tinción Azul tripan y Giemsa (Didion et al., 1989). La lectura se realizó en microscopio óptico con aumento de 1000x. Se consideraron espermatozoides vivos con acrosoma intacto los que presentaron un co- loración pálida o transparente en la parte posterior a la línea ecuatorial de la cabeza y al mismo tiempo tenían el acrosoma teñido de color fucsia.

La capacitación espermática fue evaluada en forma indirecta mediante la inducción de la exocitosis acrosomal utilizando ionóforo de calcio A23187 (Sigma C-7522) (De las Heras et al., 1997) en el grupo sin antioxidante y en el que se obtuvo los mejores resultados con el uso de antioxidantes. Las muestras fueron incubadas por $15 \mathrm{~min}$ en la presencia de $5 \mathrm{mM}$ de calcio ionóforo A23187 a $37^{\circ} \mathrm{C}$. Luego se realizó la técnica de doble tinción descrita anteriormente. Los espermatozoides que presentaron su acrosoma sin teñir con Giemsa y que tenían una coloración pálida o transparente en la cabeza fueron considerados espermatozoides reaccionados.

\section{Análisis estadístico}

Se usó el análisis de varianza para evaluar el efecto de los tratamientos sobre los porcentajes de motilidad, integridad del acrosoma, y viabilidad y capacitación espermática. La prueba de Tukey fue utilizada para determinar diferencias significativas entre grupos (Zar, 1999). Los porcentajes fueron transformados a valores angulares (ángulo = arco seno $?$ porcentaje) para acercar los datos a la distribución normal. Se utilizó el programa estadístico Prism® v. 3.0.

Para uniformizar los valores iniciales del semen fresco de cada ensayo, los porcentajes de motilidad progresiva y viabilidad e integridad del acrosoma se expresaron como las proporciones entre los valores inmediatamente descongelados con los valores del semen fresco (Aisen et al., 2000).

\section{Resultados y Discusión}

La adición de Tempo 0.5 y $1.0 \mathrm{mM}$ mantuvieron la motilidad progresiva postdescongelamiento; sin embargo, ninguna de 
Cuadro 1. Efecto de la concentración de Tempo y Tempol en la calidad del semen ovino post-descongelamiento

\begin{tabular}{|c|c|c|}
\hline Tratamientos & Motilidad progresiva & $\begin{array}{c}\text { Viabilidad e integridad } \\
\text { acrosomal }\end{array}$ \\
\hline Sin antioxidante & $66.9 \pm 4.81^{\mathrm{b}}$ & $58.4 \pm 5.6^{\mathrm{b}}$ \\
\hline \multicolumn{3}{|l|}{ Tempo } \\
\hline $0.5 \mathrm{mM}$ & $78.9 \pm 4.7^{\mathrm{a}}$ & $69.9 \pm 6.4^{\mathrm{a}}$ \\
\hline $1.0 \mathrm{mM}$ & $66.1 \pm 8.0^{\mathrm{b}}$ & $57.5 \pm 6.7^{b}$ \\
\hline $2.5 \mathrm{mM}$ & $48.9 \pm 4.5^{\mathrm{c}}$ & $43.8 \pm 5.0^{c}$ \\
\hline \multicolumn{3}{|l|}{ Tempol } \\
\hline $0.5 \mathrm{mM}$ & $45.1 \pm 5.7^{\mathrm{c}}$ & $40.4 \pm 5.8^{c}$ \\
\hline $1.0 \mathrm{mM}$ & $34.5 \pm 4.7^{\mathrm{d}}$ & $31.8 \pm 4.9^{\mathrm{d}}$ \\
\hline $2.5 \mathrm{mM}$ & $21.7 \pm 5.0^{\mathrm{e}}$ & $22.7 \pm 3.6^{\mathrm{e}}$ \\
\hline
\end{tabular}

Los valores son promedios de los porcentajes \pm desviación estándar

$a, b, c, d, e$ indican diferencias significativas dentro de columnas $(p<0.05)$.

El valor promedio de la motilidad progresiva y de la viabilidad e integridad acrosomal del semen fresco fue de 83.1 y $87.8 \%$, respectivamente

las concentraciones utilizadas de Tempol tuvo un efecto positivo (Cuadro 1).

Estos resultados son similares a los reportados por Santiani (2003), quien menciona que concentraciones entre 0.5 a $1.0 \mathrm{mM}$ de Tempo en un medio en base a leche descremada previenen la pérdida de motilidad progresiva post-descongelamiento, mientras que la adición de Tempol no tuvo ningún efecto sobre la motilidad de semen conservado. Por otro lado, en espermatozoides bovinos, Foote et al. (2002) señalan que ni Tempo ni Tempol en concentraciones de $0.2-2.0 \mathrm{mM}$ en un medio en base a Tris, tienen efecto y cuando se utiliza leche descremada como medio de dilución se observaron efectos negativos en la motilidad progresiva. Esto indica que podría existir diferencias entre especies y a la vez debido al dilutor base. El resultado del presente trabajo indica que el Tempo $0.5 \mathrm{mM}$ previene parcialmente los efectos dañinos de las especies reactivas de oxígeno sobre la motilidad espermática durante el pro- ceso de criopreservación, mientras que mayores concentraciones de Tempo $(2.5 \mathrm{mM})$ o la utilización de Tempol tienen efectos adversos.

En relación a la viabilidad espermática e integridad del acrosoma (Cuadro 1), los valores obtenidos siguieron la misma tendencia descrita para la motilidad espermática, siendo significativamente mayor $(\mathrm{p}<0.05)$ el porcentaje de espermatozoides vivos con acrosoma intacto, en el grupo Tempo $0.5 \mathrm{mM}$ con respecto a los demás grupos $(\mathrm{p}<0.05)$. En forma similar, Santiani (2003) encontró el mejor porcentaje de espermatozoides vivos con acrosoma intacto utilizando concentraciones entre 0.5 a $1.0 \mathrm{mM}$ de Tempo utilizando un dilutor en base a leche descremada, aunque sin diferencias estadísticas. La disminución de la viabilidad e integridad acrosomal durante el proceso de criopreservación estaría siendo inducido parcialmente por las especies reactivas de oxígeno producida durante el enfriamiento, como ha sido 
Cuadro 2. Efecto de la adición los antioxidantes Tempo y Tempol en dosis de $0.5 \mathrm{mM}$ sobre la condición del acrosoma de espermatozoides viables de ovino luego del proceso de congelamiento-descongelamiento

\begin{tabular}{lccc}
\hline $\begin{array}{c}\text { Condición del acrosoma } \\
\text { de los espermatozoides } \\
\text { vivos }\end{array}$ & Sin antioxidante & Tempo & Tempol \\
\hline Intacto & $84.9 \pm 3.8^{\mathrm{b}}$ & $91.5 \pm 4.0^{\mathrm{a}}$ & $87.4 \pm 5.2^{\mathrm{b}}$ \\
Reaccionado & $15.1 \pm 3.8^{\mathrm{b}}$ & $8.5 \pm 4.0^{\mathrm{a}}$ & $12.6 \pm 5.2^{\mathrm{b}}$ \\
\hline
\end{tabular}

${ }^{a, b}$ Promedios con letras diferentes dentro de filas son significativamente diferentes $(p<0.05)$

descrito en ovinos (Santiani, 2003), humanos (Wang et al., 1997) y bovinos (Chatterjee y Gagnon, 2001). Por lo tanto, el efecto de las especies reactivas de oxígeno sobre la viabilidad e integridad acrosomal estaría siendo reducido por la adición de Tempo $0.5 \mathrm{mM}$ al medio de dilución durante el enfriamiento.

La evaluación de la capacitación espermática prematura se hizo en el grupo control (Cuadro 2). El grupo Tempo $0.5 \mathrm{mM}$ presentó un menor porcentaje $(\mathrm{p}<0.05)$ de reacción del acrosoma en comparación con los otros grupos, lo que podría indicar que el Tempo previene la capacitación espermática prematura al disminuir el efecto dañino de las especies reactivas de oxígeno (De Lamirande et al., 1998).

El espermatozoide ovino es muy susceptible a la acción de las especies reactivas de oxígeno. Esto se debe a que sus membranas celulares contienen grandes cantidades de ácidos grasos poliinsaturados (Jones y Mann, 1977). Así también, las membranas del espermatozoide ovino poseen menor cantidad de moléculas de colesterol que otras especies, de allí que es más propenso a sufrir capacitación espermática prematura (Davis, 1981).

El mecanismo de acción de los antioxidantes empleados en este estudio sería mediante la catálisis de la dismutación del $\mathrm{O}_{2}$ en $\mathrm{H}_{2} \mathrm{O}_{2}$ (Luo, 2001). El Tempo y Tempol aparentemente no tienen efecto sobre $\mathrm{el}_{2} \mathrm{O}_{2}$
(Offer et al., 1998), pero al oxidar $\mathrm{Fe}$ y $\mathrm{Cu}$ evitan la reacción de Fenton (Luo, 2001). De esta manera, estos antioxidantes evitarían la formación de $\mathrm{OH} \cdot$ a partir del $\mathrm{H}_{2} \mathrm{O}_{2}$ y por lo tanto bloquearían el inicio de la peroxidación lipídica.

\section{Conclusiones}

El Tempo, a una concentración de 0.5 $\mathrm{mM}$, adicionado a los $10^{\circ} \mathrm{C}$ durante el enfriamiento, previene la pérdida de motilidad y viabilidad en espermatozoides ovinos, así como bloquea el inicio de la capacitación espermática, en un medio en base a Tris.

\section{Literatura Citada}

1. Aisen E,Álvarez, H, VenturinoA, Garde J. 2000. Effect of trehalose and EDTA on cryoprotective action of ram semen diluents. Theriogenology 53: 1053-1061.

2. Aitken R, Fisher H. 1994. Reactive oxygen species generation and human spermatozoa: the balance of benefit and risk. Bioessays 16: 259-267.

3. Aitken R, Paterson M, Fisher $H$, Buckingham D, Van Duin M. 1995. Redox regulation of tyrosine phosphorylation in human spermatozoa and its role in the control of human sperm function. J Cell Sci 108: 2017-2025. 
4. Álvarez J, Storey B. 1992. Evidence for increased lipid peroxidative damage and loss of superoxide dismutase activity as a model of sublethal cryodamage to human sperm during cryopreservation. J Androl 13: 232-241.

5. Askari H,Check J, Peymer N, Bollendorf A. 1994. Effect of natural antioxidants tocopherol and ascorbic acids in maintenance of sperm activity during freeze-thaw process. Arch Androl 33: 11-15.

6. Ball B, Medina V, Gravance $C$, Baumber J. 2001. Effect of antioxidants on preservation of motility, viability and acrosomal integrity of equine spermatozoa during storage at $5{ }^{\circ} \mathrm{C}$. Theriogenology 56: 577-589.

7. Bilodeau J, Blanchette S, Cormier N, Sirard M. 2002. Reactive oxygen species-mediated loss of bovine sperm motility in egg yolk Tris extender. Protection by piruvate, metal chelators and bovine liver or oviductal fluid catalase. Theriogenology 57: 1105-1122.

8. Chatterjee S, Gagnon C. 2001. Production of reactive species by spermatozoa undergoing cooling, freezing and thawing. Molecular Reprod Dev 59: 451-458.

9. Davis B. 1981. Timing of fertilization in mammals: Sperm cholesterol/phospho-lipid ratio as a determinant of the capacitation interval. Cell Biol 78: 7560-7564.

10. De Lamirande E, Tsai C, Harakat A, Gagnon C. 1998. Involve-ment of reactive oxygen species in human sperm acrosome reaction induced by A23187, lysophosphatidylcholine, and biological fluid ultrafiltrates. J Androl 19: 585-594.

11. De Las Heras M, Valcárcel A, Pérez L. 1997. In vitro capacitating effect of gamma-aminobutyric in ram spermatozoa. Biol Reprod 56: 964-968.

12. Didion B, Dabrinsky J, Giles J, Graves C. 1989. Staining procedure to detect viability and the true acrosome reaction in spermatozoa of various species. Gamete Research 22: 51-57.
13. Donoghue, A.; D. Donoghue. 1997. Effects of water- and lipid-soluble antioxidants on turkey sperm viability, membrane integrity, and motility during liquid storage. Poultry Sci 76: 1440-1445.

14. Foote R, Brockett C, Kaproth M. 2002. Motility and fertility of bull sperm in whole milk extender containing antioxidants. Anim Reprod Sci 71: 13-23.

15. Jones R, Mann T. 1977. Damage to ram spermatozoa by peroxidation of endogenous phospholipids. J Reprod Fertil 50: 261-268.

16. Lио J. 2001. Nitroxides-metalindependent SOD mimics. Free radicals in biology and medicine. Free radical and radiation biology program. USA: University of Iowa. p 745-762.

17. Mara L, Accardo C, Pilichi S, Dattena $M$, Chessa F, Chessa B, Branca A, Cappai P. 2005. Benefits of Tempol on ram semen motility and in vitro fertility: a preliminary study. Theriogenology 63 : 2243-2253.

18. Mitchell J, Samuni A, Krishna MJ, Degratt W, Ahn M, Samuni U, Russo A. 1990. Biologically active metalindependent superoxide dismutase mimics. Biochemistry 29: 2802-2807.

19. Ochsendorf F, Thiele J, Fuchs J. 1997. Antioxidants in germinal epithelium, spermatozoa and seminal plasma. In: Ochsendorf FR, Fuchs J (eds). Oxidative stress in male infertility. Germany: Michael Itschert, Gardez Verlag. p 85-128.

20. Offer T, Mohsen M, Samuni A. 1998. An SOD-mimicry mechanism underlies the role of nitroxides in protecting papain from oxidative inactivation. Free Racial Biology and Medicine 25: 832-838.

21. Sánchez-Partida L, Setchell B, Maxwell W. 1997. Epididymal compounds and antioxidants in diluents for the frozen storage of ram spermatozoa. Reprod Fertil Devel 9: 689-696.

22. Sandoval R. 2005. Criopreservación de semen ovino empleando diferentes dilutores y combinaciones de agentes 
crioprotectores permeantes y no permeantes. Tesis de Médico Veterinario. Lima: Facultad de Medicina Veterinaria, Univ. Nacional Mayor de San Marcos. 72 p.

23. Santiani A. 2003. Criopreservación de semen ovino: efecto de la adición de antioxidantes al diluyente. Tesis de Maestría en Ciencias. Temuco, Chile: Facultad de Medicina, Universidad de La Frontera. $95 \mathrm{p}$.

24. Upreti G, Jensen K, Munday $R$, Duganzich D, Vishwanath R, Smith J. 1998. Studies on aromatic amino acid oxidase activity in ram spermatozoa: role of piruvate as antioxidant. Anim Reprod Sci 51: 275-287.
25. Wang A, Zhang H, Ikemoto I, Anderson D, Loughlin K. 1997. Reactive oxygen species generation by seminal cells during cryopreservation. Urology 49: 921-925.

26. Watson P. 2000. The causes of reduced fertility with cryopreserved semen. Anim Reprod Sci 60-61: 481-492.

27. Watson P, Anderson W. 1983. Influence of butylated hydroxytoluene (BHT) on the viability of ram spermatozoa undergoing cold shock. J Reprod Fertil 69: 229-235.

28. Zar J. 1999. Biostatistical analysis. $4^{\text {th }}$ ed. New Jersey: Prentice Hall. 423 p. 